\title{
A New Conjugate Gradient Projection Method for Solving Stochastic Generalized Linear Complementarity Problems
}

\author{
Zhimin Liu, Shouqiang Du, Ruiying Wang \\ College of Mathematics and Statistics, Qingdao University, Qingdao, China \\ Email: 1475435458@qq.com, sqdu@qdu.edu.cn,694293620@qq.com
}

Received 2 May 2016; accepted 10 June 2016; published 13 June 2016

Copyright (C) 2016 by authors and Scientific Research Publishing Inc.

This work is licensed under the Creative Commons Attribution International License (CC BY). http://creativecommons.org/licenses/by/4.0/

(c) (i) Open Access

\section{Abstract}

In this paper, a class of the stochastic generalized linear complementarity problems with finitely many elements is proposed for the first time. Based on the Fischer-Burmeister function, a new conjugate gradient projection method is given for solving the stochastic generalized linear complementarity problems. The global convergence of the conjugate gradient projection method is proved and the related numerical results are also reported.

\section{Keywords}

Stochastic Generalized Linear Complementarity Problems, Fischer-Burmeister Function, Conjugate Gradient Projection Method, Global Convergence

\section{Introduction}

Suppose $\left(\Omega_{1}, F, G, P\right)$ is a probability space with $\Omega_{1} \subseteq \mathfrak{R}^{n} ; P$ is a known probability distribution. The stochastic generalized linear complementarity problems (denoted by SGLCP) is to find $x \in \mathfrak{R}^{n}$, such that

$$
F(x, \omega):=M_{1}(\omega) x+q_{1}(\omega) \geq 0, G(x, \omega):=M_{2}(\omega) x+q_{2}(\omega) \geq 0, F^{\mathrm{T}}(x, \omega) G(x, \omega)=0,
$$

where $M_{1}(\omega), M_{2}(\omega) \in \mathfrak{R}^{n \times n}$ and $q_{1}(\omega), q_{2}(\omega) \in \mathfrak{R}^{n}$ for $\omega \in \Omega_{1}$, are random matrices and vectors. When $G(x, \omega)=x$, stochastic generalized linear complementarity problems reduce to the classic Stochastic Linear Complementarity Problems (SLCP), which has been studied in [1]-[7]. Generally, they usually apply the Expected Value (EV) method and Expected Residual Minimization (ERM) method to solve this kind of problem.

If $\Omega_{1}$ only contains a single realization, then (1) reduces to the following standard Generalized Linear Complementarity Problem (GLCP), which is to find a vector $x \in \Re^{n}$ such that 


$$
F(x):=M_{1} x+q_{1} \geq 0, G(x):=M_{2} x+q_{2} \geq 0, F^{\mathrm{T}}(x) G(x)=0,
$$

where $M_{1}, M_{2} \in \mathfrak{R}^{n \times n}$ and $q_{1}, q_{2} \in \mathfrak{R}^{n}$.

In this paper, we consider the following generalized stochastic linear complementarity problems. Denote $\Omega_{1}=\left\{\omega_{1}, \omega_{2}, \cdots, \omega_{m}\right\}$, to find an $x \in \mathfrak{R}^{n}$ such that

$$
\begin{aligned}
& F\left(x, \omega_{i}\right):=M_{1}\left(\omega_{i}\right) x+q_{1}\left(\omega_{i}\right) \geq 0, \\
& G\left(x, \omega_{i}\right):=M_{2}\left(\omega_{i}\right) x+q_{2}\left(\omega_{i}\right) \geq 0, \quad i=1, \cdots, m, m>1 . \\
& F^{\mathrm{T}}\left(x, \omega_{i}\right) \cdot G\left(x, \omega_{i}\right)=0 .
\end{aligned}
$$

Let $\bar{M}_{j}=\sum_{i=1}^{m} p_{i} M_{j}\left(\omega_{i}\right), \bar{q}_{j}=\sum_{i=1}^{m} p_{i} q_{j}\left(\omega_{i}\right)$, where $p_{i}=P\left(\omega_{i} \in \Omega_{1}\right)>0, i=1, \cdots, m, j=1,2$. Then (2) is equivalent to (3) and (4)

$$
\begin{aligned}
\bar{M}_{1} x+\bar{q}_{1} \geq 0, \bar{M}_{2} x+\bar{q}_{2} \geq 0,\left(\bar{M}_{1} x+\bar{q}_{1}\right)^{\mathrm{T}} \cdot\left(\bar{M}_{2} x+\bar{q}_{2}\right)=0, \\
\\
M_{1}\left(\omega_{i}\right) x+q_{1}\left(\omega_{i}\right) \geq 0, \\
M_{2}\left(\omega_{i}\right) x+q_{2}\left(\omega_{i}\right) \geq 0, \quad i=1, \cdots, m .
\end{aligned}
$$

In the following of this paper, we consider to give a new conjugate gradient projection method for solving (2). The method is based on a suitable reformulation. Base on the Fischer-Burmeister function, $x$ is a solution of (3) $\Leftrightarrow \phi(x)=0$, where

$$
\phi(x)=\left(\begin{array}{c}
\phi\left(\left(\bar{M}_{1} x+\bar{q}_{1}\right)_{1},\left(\bar{M}_{2} x+\bar{q}_{2}\right)_{1}\right) \\
\vdots \\
\phi\left(\left(\bar{M}_{1} x+\bar{q}_{1}\right)_{n},\left(\bar{M}_{2} x+\bar{q}_{2}\right)_{n}\right)
\end{array}\right)
$$

Define

$$
\Psi(x)=\frac{1}{2}\|\phi(x)\|^{2}
$$

Then solving (3) is equivalent to find a global solution of the minimization problem

$$
\min _{x \in \Re^{n}} \Psi(x) .
$$

So, (3) and (4) can be rewritten as

$$
H(x, y)=0, y \geq 0,
$$

where

$$
H(x, y)=\left(\begin{array}{c}
\phi(x) \\
M_{1}\left(\omega_{1}\right) x+q_{1}\left(\omega_{1}\right)-y_{1} \\
\vdots \\
M_{1}\left(\omega_{m}\right) x+q_{1}\left(\omega_{m}\right)-y_{m} \\
M_{2}\left(\omega_{1}\right) x+q_{2}\left(\omega_{1}\right)-y_{m+1} \\
\vdots \\
M_{2}\left(\omega_{m}\right) x+q_{2}\left(\omega_{m}\right)-y_{2 m}
\end{array}\right),
$$

$y=\left[y_{1}^{\mathrm{T}}, y_{2}^{\mathrm{T}}, \cdots, y_{2 m}^{\mathrm{T}}\right]^{\mathrm{T}} \in \mathfrak{R}^{2 m \times n}$ is slack variable with $y_{i} \in \mathfrak{R}^{n}, \quad i=1, \cdots, 2 m$.

Let $x=x^{\prime}-x^{\prime \prime}$, where $x^{\prime}, x^{\prime \prime} \in \mathfrak{R}^{n}$ and $x^{\prime}, x^{\prime \prime} \geq 0$. Then we know that $H\left(x^{\prime}, x^{\prime \prime}, y\right)=0$ has $(2 m+2) n$ equations with $(2 m+2) n$ variables.

Let $t=\left(x^{\prime}, x^{\prime \prime}, y\right) \in \mathfrak{R}_{+}^{(2 m+2) n}$ and define a merit function of (5) by 


$$
\theta(t)=\frac{1}{2}\|H(t)\|^{2} .
$$

If (2) has a solution, then solving (5) is equivalent to find a global solution of the following minimization problem

$$
\begin{aligned}
& \min \theta(t) \\
& \text { s.t. } t \in \Omega
\end{aligned}
$$

where $\Omega=\left\{t \mid t \in \mathfrak{R}_{+}^{(2 m+2) n}\right\}$.

\section{Preliminaries}

In this section, we give some Lemmas, which are taken from [8]-[10].

Lemma 1. Let $P$ be the projection onto $\Omega$, let $t(s)=P(t+s)$ for given $t \in \Omega$ and $s \in \mathfrak{R}^{(2 m+2) n}$, then 1) $\langle t(s)-(t+s), y-t(s)\rangle \geq 0$, for all $y \in \Omega$.

2) $P$ is a non-expansive operator, that is, $\|P(y)-P(x)\| \leq\|y-x\|$ for all $x, y \in \mathfrak{R}^{(2 m+2) n}$.

3) $\langle-s, t-t(s)\rangle \geq\|t(s)-t\|^{2}$.

Lemma 2. Let $\nabla_{\Omega} \theta(t)$ be the projected gradient of $\theta$ at $t \in \Omega$.

1) $\min \{\nabla \theta(t), v: v \in T(t),\|v\| \leq 1\}=-\left\|\nabla_{\Omega} \theta(t)\right\|$.

2) The mapping $\left\|\nabla_{\Omega} \theta(\cdot)\right\|$ is lower semicontinuous on $\Omega$, that is, if $\lim _{k \rightarrow \infty} t_{k} \rightarrow t$, then

$$
\left\|\nabla_{\Omega} \theta(t)\right\| \leq \liminf _{k \rightarrow \infty}\left\|\nabla_{\Omega} \theta\left(t_{k}\right)\right\| .
$$

3) The point $t^{*} \in \Omega$ is a stationary point of problem (6) $\Leftrightarrow \nabla_{\Omega} \theta\left(t^{*}\right)=0$.

\section{The Conjugate Gradient Projection Method and Its Convergence Analysis}

In this section, we give a new conjugate gradient projection method and give some discussions about this method.

Given an iterate $t_{k} \in \Omega=\left\{t \mid t \in \mathfrak{R}_{+}^{(2 m+2) n}\right\}$, we let $\overline{t_{k}}\left(s_{k}\right)=P\left[t_{k}-\nabla \theta\left(t_{k}\right)\right]$,

$$
t_{k+1}=t_{k}\left(s_{k}\right)=P\left[t_{k}+s_{k}\right] \text {, }
$$

where $s_{k}=\left\{\begin{array}{ll}-\nabla \theta\left(t_{k}\right) & k=1 \\ -\nabla \theta\left(t_{k}\right)+\beta_{k} d_{k-1} & k>1\end{array}\right.$. Inspired by the literature [8]-[11], we take

$$
\left|\beta_{k}\right|=\frac{\left\|\bar{t}_{k}\left(s_{k}\right)-t_{k}\right\|^{2}}{(1+\lambda)\left\|\nabla \theta\left(t_{k}\right)\right\|\left\|d_{k-1}\right\|},
$$

with $\lambda>0$.

And $d_{k}$ is defined by

$$
d_{k}=t_{k}\left(s_{k}\right)-t_{k} .
$$

\section{Method 1. Conjugate Gradient Projection Method (CGPM)}

Step 0: Let $t_{1} \in \Omega, 0 \leq \varepsilon \leq 1, \sigma_{1}, \sigma_{2} \in(0,1), \quad \beta_{1}=0, d_{0}=0$, set $k=1$.

Step 1: Compute $\alpha_{k}$, such that

$$
\begin{gathered}
\theta\left(t_{k}+\alpha_{k} d_{k}\right) \leq \theta\left(t_{k}\right)+\sigma_{1} \alpha_{k} \nabla \theta\left(t_{k}\right)^{\mathrm{T}} d_{k}, \\
\nabla \theta\left(t_{k}+\alpha_{k} d_{k}\right)^{\mathrm{T}} d_{k} \geq \sigma_{2} \nabla \theta\left(t_{k}\right)^{\mathrm{T}} d_{k} .
\end{gathered}
$$

Set $t_{k+1}=t_{k}+\alpha_{k} d_{k}$.

Step 2: If $\left\|t_{k}-t_{k}\left(s_{k}\right)\right\| \leq \varepsilon$, stop, $t^{*}=t_{k}\left(s_{k}\right)$.

Step 3: Let $k:=k+1$, and go to Step 1 .

In order to prove the global convergence of the Method 1, we give the following assumptions. 


\section{Assumptions 1}

1) $\theta(t)$ has a lower bound on the level set $L_{0}=\left\{t_{1} \in \mathfrak{R}^{(2 m+2) n} \mid \theta(t) \leq \theta\left(t_{1}\right)\right\}$, where $t_{1}$ is initial point.

2) $\theta(t)$ is continuously differentiable on the $L_{0}$, and its gradient is Lipschitz continuous, that is, there exists a positive constant $L$ such that

$$
\|g(u)-g(v)\| \leq L\|u-v\| \quad \forall u, v \in L_{0} .
$$

Lemma 3. If $t_{k}$ is not the stability point of (6), $t_{k} \neq t_{k}\left(s_{k}\right)$, then search direction $d_{k}$ generated by (9) descent direction, which is $\left\langle\nabla \theta\left(t_{k}\right), d_{k}\right\rangle \leq-\frac{\lambda}{1+\lambda}\left\|\nabla \theta\left(t_{k}\right)\right\|^{2}<0$.

Proof. From (7), Lemma 1, and (8), we have

$$
\begin{aligned}
& \left\langle\nabla \theta\left(t_{k}\right), d_{k}\right\rangle=\left\langle\nabla \theta\left(t_{k}\right), t_{k}\left(s_{k}\right)-t_{k}\right\rangle \\
& =\left[\left\langle\nabla \theta\left(t_{k}\right), t_{k}\left(s_{k}\right)-\overline{t_{k}}\left(s_{k}\right)\right\rangle+\left\langle\nabla \theta\left(t_{k}\right), \overline{t_{k}}\left(s_{k}\right)-t_{k}\right\rangle\right] \\
& \leq\left\|\nabla \theta\left(t_{k}\right)\right\|\left\|t_{k}\left(s_{k}\right)-\overline{t_{k}}\left(s_{k}\right)\right\|-\left\langle\nabla \theta\left(t_{k}\right), t_{k}-\overline{t_{k}}\left(s_{k}\right)\right\rangle \\
& \leq \mid \beta_{k}\left\|\nabla \theta\left(t_{k}\right)\right\|\left\|d_{k-1}\right\|-\left\|\overline{t_{k}}\left(s_{k}\right)-t_{k}\right\|^{2} \\
& \leq\left(\frac{1}{1+\lambda}-1\right)\left\|\overline{t_{k}}\left(s_{k}\right)-t_{k}\right\|^{2} \\
& \leq \frac{-\lambda}{1+\lambda}\left\|\nabla \theta\left(t_{k}\right)\right\|^{2}<0 .
\end{aligned}
$$

Lemma 4. [11] Suppose that Assumptions 1 holds. Let $\theta(t)$ continuously differentiable and lower bound on the $\Omega, \nabla \theta(t)$ is uniformly continuous on the $\Omega$ and $\left\{\nabla \theta\left(t_{k}\right)\right\}$ is bounded, then $\left\{t_{k}\right\}$ generated by Method 1 are satisfied

$$
\lim _{k \rightarrow \infty}\left\|t_{k}-t_{k}\left(s_{k}\right)\right\|=0, \lim _{k \rightarrow \infty}\left\|t_{k}-\overline{t_{k}}\left(s_{k}\right)\right\|=0 .
$$

Theorem 1. Let $\theta(t)$ continuously differentiable and lower bound on the $\Omega, \nabla \theta(t)$ is uniformly continuous on the $\Omega,\left\{t_{k}\right\}$ is a sequence generated by Method 1 , then $\lim _{k \rightarrow \infty}\left\|\nabla_{\Omega} \theta\left(t_{k}\right)\right\|=0$, and any accumulation point of $\left\{t_{k}\right\}$ is a stationary point of (6).

Proof. By Lemma 2, we have $\forall \varepsilon>0, \exists v_{k} \in T_{\Omega}\left(t_{k}\right),\left\|v_{k}\right\| \leq 1$, satisfy

$$
\left\|\nabla_{\Omega} \theta\left(t_{k}\right)\right\| \leq\left\langle-\nabla \theta\left(t_{k}\right), v_{k}\right\rangle+\varepsilon,
$$

for $\forall z \in \Omega$, by Lemma 1 , we know that $\left\langle t_{k}\left(s_{k}\right)-\left(t_{k}+s_{k}\right), z-t_{k}\left(s_{k}\right)\right\rangle \geq 0$, and we have $\left\langle s_{k}, z-t_{k}\left(s_{k}\right)\right\rangle \leq\left\langle t_{k}\left(s_{k}\right)-t_{k}, z-t_{k}\left(s_{k}\right)\right\rangle \leq\left\|t_{k}\left(s_{k}\right)-t_{k}\right\|\left\|z-t_{k}\left(s_{k}\right)\right\|$, so,

$$
\left\langle s_{k}, z-t_{k}\left(s_{k}\right)\right\rangle \leq\left\|t_{k}\left(s_{k}\right)-t_{k}\right\|\left\|z-t_{k}\left(s_{k}\right)\right\| .
$$

Let $v_{k+1}=z-t_{k}\left(s_{k}\right) \in T_{\Omega}\left(t_{k+1}\right),\left\|v_{k+1}\right\| \leq 1$, from (11), we have

$$
\left\langle s_{k}, v_{k+1}\right\rangle=\left\langle-\nabla \theta\left(t_{k}\right)+\beta_{k} d_{k-1}, v_{k+1}\right\rangle \leq\left\|t_{k}\left(s_{k}\right)-t_{k}\right\| .
$$

By the above formula, (8) and Lemma 1, we get

$$
\begin{aligned}
\left\langle-\nabla \theta\left(t_{k}\right), v_{k+1}\right\rangle & \leq\left\|t_{k}\left(s_{k}\right)-t_{k}\right\|+\left|\beta_{k}\right|\left\|d_{k-1}\right\| \\
& \leq\left\|t_{k}\left(s_{k}\right)-t_{k}\right\|+\frac{1}{(1+\lambda)\left\|\nabla \theta\left(t_{k}\right)\right\|}\left\|\overline{t_{k}}\left(s_{k}\right)-t_{k}\right\|^{2} \\
& \leq\left\|t_{k}\left(s_{k}\right)-t_{k}\right\|+\frac{1}{1+\lambda}\left\|t_{k}\left(s_{k}\right)-t_{k}\right\| .
\end{aligned}
$$

Taking limit on both sides and by Lemma 4 , we know that 


$$
\lim _{k \rightarrow \infty} \sup \left\langle-\nabla \theta\left(t_{k}\right), v_{k+1}\right\rangle=0 .
$$

Because

$$
\begin{aligned}
\left\langle-\nabla \theta\left(t_{k}\left(s_{k}\right)\right), v_{k+1}\right\rangle & =\left\langle\nabla \theta\left(t_{k}\right)-\nabla \theta\left(t_{k}\left(s_{k}\right)\right), v_{k+1}\right\rangle+\left\langle-\nabla \theta\left(t_{k}\right), v_{k+1}\right\rangle \\
& \leq\left\|\nabla \theta\left(t_{k}\right)-\nabla \theta\left(t_{k}\left(s_{k}\right)\right)\right\|+\left\langle-\nabla \theta\left(t_{k}\right), v_{k+1}\right\rangle
\end{aligned}
$$

and Lemma 4, we have

$$
\lim _{k \rightarrow \infty}\left\|t_{k}-t_{k}\left(s_{k}\right)\right\|=0 .
$$

By (12), (13), (14) and $\nabla \theta(t)$ is uniformly continuous on the $\Omega$, we get

$$
\lim _{k \rightarrow \infty} \sup \left\langle-\nabla \theta\left(t_{k}\left(s_{k}\right)\right), v_{k+1}\right\rangle=0 .
$$

By (10), we know that

$$
\lim _{k \rightarrow \infty}\left\|\nabla_{\Omega} \theta\left(t_{k}\right)\right\|=0 .
$$

Let $\lim _{k \in N_{0}, k \rightarrow \infty} t_{k}=t$, where $N_{0} \subseteq N$, by Lemma 2 and (15), we have

$$
\left\|\nabla_{\Omega} \theta(t)\right\| \leq \lim _{k \in N_{0}, k \rightarrow \infty} \inf \left\|\nabla_{\Omega} \theta\left(t_{k}\right)\right\|=0 .
$$

From Lemma 23 ), we get any accumulation point of $\left\{t_{k}\right\}$ is a stationary point of (6).

\section{Numerical Results}

In this section, we give the numerical results of the conjugate gradient projection method for the following given test problems, which are all given for the first time. We present different initial point $t_{0}$, which indicates that Method 1 is global convergence.

Throughout the computational experiments, according to Method 1 for determining the parameters, we set the parameters as

$$
\sigma_{1}=0.49, \sigma_{2}=0.5, \lambda=1.067 .
$$

The stopping criterion for the method is $\left\|g_{k}\right\| \leq 10^{-6}$ or $k_{\max }=100000$.

In the table of the test results, $t_{0}$ denotes initial point, $x^{*}$ denotes the solution, val denotes the final value of $\theta(t)=\frac{1}{2}\|H(t)\|^{2}$, Itr denotes the number of iteration.

Example 1. Considering SGLCP with

$$
\begin{gathered}
M_{1}(\omega)=\left(\begin{array}{ccc}
\frac{3}{2}+\omega & -1 & 0 \\
-1 & \frac{3}{2}+\omega & -1 \\
0 & -1 & \frac{3}{2}+\omega
\end{array}\right), q_{1}(\omega)=\left(\begin{array}{l}
\frac{1}{2}+\omega \\
\frac{1}{2}+\omega \\
\frac{1}{2}+\omega
\end{array}\right), \\
M_{2}(\omega)=\left(\begin{array}{ccc}
\frac{5}{2}+\omega & -1 & 0 \\
-1 & \frac{5}{2}+\omega & -1 \\
0 & -1 & \frac{5}{2}+\omega
\end{array}\right), q_{2}(\omega)=\left(\begin{array}{l}
1+\omega \\
1+\omega \\
1+\omega
\end{array}\right), \\
\Omega_{1}=\left\{\omega_{1}, \omega_{2}\right\}=\{0,1\} \text { and } p_{i}=P\left(\omega_{i} \in \Omega_{1}\right)=0.5, i=1,2 .
\end{gathered}
$$

The test results are listed in “Table 1" using different initial points. 
Table 1. Results of the numerical Example 1-2 using method 1.

\begin{tabular}{ccccc}
\hline Problem & $t_{0}$ & $x^{*}$ & val & Itr \\
\hline & $0.5 \times(1,1, \cdots, 1)$ & $(-0.8385,-1.0548,-0.8385)$ & $3.3 \times 10^{-3}$ & 1465 \\
& $(1,1, \cdots, 1)$ & $(-0.8385,-1.0548,-0.8385)$ & $3.3 \times 10^{-3}$ & 1701 \\
Example 1 & $5 \times(1,1, \cdots, 1)$ & $(-0.8385,-1.0548,-0.8385)$ & $3.3 \times 10^{-3}$ & 2670 \\
& $10 \times(1,1, \cdots, 1)$ & $(-0.8385,-1.0548,-0.8385)$ & $3.3 \times 10^{-3}$ & 3261 \\
& $20 \times(1,1, \cdots, 1)$ & $(-0.8385,-1.0548,-0.8385)$ & $3.3 \times 10^{-3}$ & 3847 \\
& $50 \times(1,1, \cdots, 1)$ & $(-0.8385,-1.0548,-0.8385)$ & $3.3 \times 10^{-3}$ & 4704 \\
\hline & $0.5 \times(1,1, \cdots, 1)$ & $(-0.3747,0.1516,-0.0276,-0.0770,0.2306,-0.9539,1.4488)$ & 0.7299 & 62788 \\
& $(1,1, \cdots, 1)$ & $(-0.3746,0.1516,-0.0276,-0.0770,0.2306,-0.9539,1.4488)$ & 0.7299 & 65528 \\
& $5 \times(1,1, \cdots, 1)$ & $(-0.3746,0.1516,-0.0276,-0.0770,0.2306,-0.9539,1.4488)$ & 0.7299 & 66962 \\
& $10 \times(1,1, \cdots, 1)$ & $(-0.3746,0.1516,-0.0276,-0.0770,0.2306,-0.9539,1.4488)$ & 0.7299 & 100,000 \\
& $20 \times(1,1, \cdots, 1)$ & $(-0.3746,0.1516,-0.0276,-0.0770,0.2306,-0.9539,1.4488)$ & 0.7299 & 100,000 \\
& $50 \times(1,1, \cdots, 1)$ & $(-0.3746,0.1516,-0.0276,-0.0770,0.2306,-0.9539,1.4488)$ & 0.7299 & 100,000 \\
\hline
\end{tabular}

Example 2. Considering SGLCP with

$$
M_{1}(\omega)=\left(\begin{array}{ccccccc}
\frac{1}{2}+\omega & 2 & 2 & 2 & 2 & 2 & 2 \\
0 & \frac{1}{2}+\omega & 2 & 2 & 2 & 2 & 2 \\
0 & 0 & \frac{1}{2}+\omega & 2 & 2 & 2 & 2 \\
0 & 0 & 0 & \frac{1}{2}+\omega & 2 & 2 & 2 \\
0 & 0 & 0 & 0 & \frac{1}{2}+\omega & 2 & 2 \\
0 & 0 & 0 & 0 & 0 & \frac{1}{2}+\omega & 2 \\
0 & 0 & 0 & 0 & 0 & 0 & \frac{1}{2}+\omega
\end{array}\right), \quad \begin{gathered}
-\frac{3}{2}+\omega \\
-\frac{3}{2}+\omega \\
-\frac{3}{2}+\omega \\
3 \\
0
\end{gathered}
$$




$$
\Omega_{1}=\left\{\omega_{1}, \omega_{2}\right\}=\{0,1\} \text { and } p_{i}=P\left(\omega_{i} \in \Omega_{1}\right)=0.5, \quad i=1,2 .
$$

The test results are listed in "Table 1" using different initial points.

\section{Conclusion}

In this paper, we present a new conjugate gradient projection method for solving stochastic generalized linear complementarity problems. The global convergence of the method is analyzed and numerical results show that Method 1 is effective. In future work, large-scale stochastic generalized linear complementarity problems need to be studied and developed.

\section{Acknowledgements}

This work is supported by National Natural Science Foundation of China (No. 11101231, 11401331), Natural Science Foundation of Shandong (No. ZR2015AQ013) and Key Issues of Statistical Research of Shandong Province (KT15173).

\section{References}

[1] Chen, X. and Fukushima, M. (2005) Expected Residual Minimization Method for Stochastic Linear Complementarity Problems. Mathematics of Operations Research, 30, 1022-1038. http://www-optima.amp.i.kyoto-u.ac.jp/ fuku/papers/SLCP-MOR-rev.pdf http://dx.doi.org/10.1287/moor.1050.0160

[2] Chen, X., Zhang, C. and Fukushima, M. (2009) Robust Solution of Monotone Stochastic Linear Complementarity Problems. Mathematical Programming, 117, 51-80. http://link.springer.com/article/10.1007/s10107-007-0163-Z http://dx.doi.org/10.1007/s10107-007-0163-z

[3] Lin, G.H. and Fukushima, M. (2006) New Reformulations for Stochastic Nonlinear Complementarity Problems. Optimization Methods and Software, 21, 551-564.

http://web.a.ebscohost.com/ehost/detail/detail?sid=beded7da-701c-4790-b1c9-81d20182cd04\%40sessionmgr4005\&vid =0\&hid=4201\&bdata=Jmxhbmc9emgtY24mc210ZT1laG9zdC1saXZl\&preview=false\#AN=22089195\&db=aph http://dx.doi.org/10.1080/10556780600627610

[4] Lin, G.H., Chen, X. and Fukushima, M. (2010) New Restricted NCP Functions and Their Applications to Stochastic NCP and Stochastic MPEC. Optimization, 56, 641-653. http://www.amp.i.kyoto-u.ac.jp/tecrep/ps_file/2006/2006-011.pdf http://dx.doi.org/10.1080/02331930701617320

[5] Ling, C., Qi, L., Zhou, G. and Caccetta, L. (2008) The SC 1 Property of an Expected Residual Function Arising from Stochastic Complementarity Problems. Operations Research Letters, 36, 456-460. http://espace.library.curtin.edu.au/cgi-bin/espace.pdf?file=/2009/07/20/file_27/119233 http://dx.doi.org/10.1016/j.orl.2008.01.010

[6] Fang, H.T., Chen, X.J. and Fukushima, M. (2007) Stochastic $\mathfrak{R}_{0}$ Matrix Linear Complementarity Problems. SIAM Journal on Optimization, 18, 482-506. http://www.polyu.edu.hk/ama/staff/xjchen/SIOPT_FCF.pdf http://dx.doi.org/10.1137/050630805

[7] Gürkan, G., Ozge, A.Y. and Robinson, S.M. (1999) Sample-Path Solution of Stochastic Variational Inequalities. Mathematical Programming, 84, 313-333. http://link.springer.com/article/10.1007/s101070050024 http://dx.doi.org/10.1007/s101070050024

[8] Sun, Q.Y., Wang, C.Y. and Shi, Z.J. (2006) Global Convergence of a Modified Gradient Projection Method for Convex Constrained Problems. Acta Mathematicale Applicatae Sinica, 22, 227-242. http://link.springer.com/article/10.1007/s10255-006-0299-2 http://dx.doi.org/10.1007/s10255-006-0299-2

[9] Wang, C.Y. and Qu, B. (2002) Convergence of the Gradient Projection Method with a New Stepsize Rule. Operations Research Transactions, 6, 36-44. http://www.cnki.net/KCMS/detail/detail.aspx?QueryID=0\&CurRec=4\&recid=\&filename=YCXX200201004\&dbname =CJFD2002\&dbcode=CJFQ\&pr=\&urlid=\&yx=\&v=MDM0OTdJUjhlWDFMdXhZUzdEaDFUM3FUcldNMUZyQ1V STHlmWXVadUZ5N2xWcnpJUEM3VGRyRzRIdFBNcm85Rlk

[10] Sun, Q.Y., Gao, B., Jian, L. and Wang, C.Y. (2010) Modified Conjugate Gradient Projection Method for Nonlinear Constrained Optimization. Acta Mathematicae Applicatae Sinica, 33, 640-651. 
http://d.g.wanfangdata.com.cn/Periodical_yysxxb201004008.aspx

[11] Jing, S.J. and Zhao, H.Y. (2014) Conjugate Gradient Projection Method of Constrained Optimization Problems with Wolfe Stepsize Rule. Journal of Mathematics, 34, 1193-1199.

http://qikan.cqvip.com/article/detail.aspx?id=662962703\&from=zk_search 